\title{
Approximate symmetries and conservation laws of the classical Camassa-Holm equation
}

\author{
Stylianos Dimas, $\quad$ Igor L. Freire, \\ CMCC - Centro de Matemática, Computação e Cognição, UFABC, \\ 09210 - 170, Santo André, SP \\ E-mail: s.dimas@ufabc.edu.br, $\quad$ igor.freire@ufabc.edu.br.
}

\begin{abstract}
By assuming that the arbitrary parameter involved in the celebrated Camassa-Holm equation takes very small values we study it as a perturbed PDE; we obtain its approximate symmetries and through them we construct approximate conservation laws and solutions.
\end{abstract}

Keywords: Camassa-Holm equation, approximate symmetries, approximate conservation laws, computer assisted research

\section{Introduction}

The celebrated Camassa-Holm equation,

$$
u_{t}+k u_{x}-u_{t x x}+3 u u_{x}=2 u_{x} u_{x x}+u u_{x x x}
$$

where $u$ is the fluid velocity along the $x$ direction, first appear in the classical work of Camassa and Holm as a swallow water equation derived from the Hamiltonian for Euler's equations in the swallow water regime $[4,5]$. Equation (1) possesses a large number of attributes: it is integrable, bi-Hamiltonian, admits soliton solutions and other properties characteristic of the $\mathrm{KdV}$ type of equations. This is the reason why it is one of the most well studied equations in the literature $[7,10]$. Among others it was studied by symmetry analysis methods, shown that - like the KdV equation - is self-adjoint and conservation laws were constructed $[8,12]$.

Constructing conservation laws using symmetries in an algorithmic fashion is a relative new method introduced in [14-16]. The method can be used even when the PDE do not possesses a Lagrangian or is of even order. In addition, any kind of symmetry can be used in order to construct a conservation law. Lately, this method is extended in perturbation theory using the concept of approximate symmetries $[1,3,9,17]$ in order to construct approximate conservation laws $[18,19,23]$.

In the present work we consider equation (1) as a perturbation of the PDE

$$
u_{t}-u_{t x x}+3 u u_{x}=2 u_{x} u_{x x}+u u_{x x x}
$$

i.e. we assume that the arbitrary parameter $k \ll 1$, obtain the approximate symmetries and construct its approximate conservation law. To our knowledge this is the first time that the approximate symmetries of equation (1) are given and used for obtaining an approximate conservation law for it. For all the calculations involved the symbolic package SYM for Mathematica was used [6].

In the next section we present in brief the basic notions from the symbolic arsenal used in the remainder of this paper. In section 3 the main results of this work are given. Namely, the approximate symmetries and a non trivial approximate conservation law. Finally, we briefly discuss these results on the last section. 


\section{Approximate symmetries and conservation laws}

\subsection{Approximate symmetries}

The notion of an approximate symmetry builds upon the well established in the literature notion of classical, or Lie, symmetry and its extensions $[2,11,13,20-22]$ : the symmetry condition must now be satisfied only up to the chosen order of the approximation.

Consider a system of differential equations

$$
F_{\alpha}=F_{\alpha}^{0}+\nu F_{\alpha}^{1}=0, \quad \alpha=1, \cdots, m,
$$

where $\nu$ is a small parameter (for $\nu=0$, we have the unperturbed system). An approximate Lie symmetry group of the above system is a local one-parameter group of diffeomorphisms $(x, u) \mapsto(\bar{x}(x, u, \varepsilon, \nu), \bar{u}(x, u, \varepsilon, \nu))=: T_{\varepsilon}$ such that

a) $\bar{x}(x, u, 0, \nu) \approx x, \bar{u}(x, u, 0, \nu) \approx u$

b) $T_{\varepsilon} T_{\delta}=T_{\varepsilon+\delta}$;

c) the infinitesimal generator $X$ of the approximated Lie symmetry group is

$$
X=\xi^{i}(x, u, \nu) \frac{\partial}{\partial x^{i}}+\eta^{\alpha}(x, u, \nu) \frac{\partial}{\partial u^{\alpha}},
$$

where

$$
\xi^{i}(x, u, \nu)=\left.\frac{\partial \bar{x}^{i}(x, u, \varepsilon, \nu)}{\partial \varepsilon}\right|_{\varepsilon=0}, \eta^{\alpha}(x, u, \nu)=\left.\frac{\partial \bar{\eta}^{\alpha}(x, u, \varepsilon, \nu)}{\partial \varepsilon}\right|_{\varepsilon=0} .
$$

Besides, the approximated generator (4) can readily be written as

$$
X=X_{0}+\nu X_{1}
$$

where $X_{0}$ is a Lie point symmetry generator of the unperturbed system $F_{\alpha}^{0}=0$ and $X_{1}$ is retrieved from the conditions

$$
\left.X_{1}\left(F_{\alpha}^{0}\right)\right|_{F_{\alpha}^{0}=0}+H=0
$$

and

$$
H=\left.\frac{1}{\nu} X_{0}\left(F_{\alpha}^{0}+\nu F_{\alpha}^{1}\right)\right|_{F_{\alpha}^{0}+\nu F_{\alpha}^{1}=0},
$$

where a suitable prolongation of the symmetry generators $X_{1}, X_{0}$ is assumed. Hence the approximate symmetry condition is now

$$
\left.X\left(F_{\alpha}^{0}+\nu F_{\alpha}^{1}\right)\right|_{F_{\alpha}^{0}+\nu F_{\alpha}^{1}=0}=\mathcal{O}\left(\nu^{2}\right)
$$

again a suitable prolongation for the operator (4) is assumed to be chosen.

\subsection{Approximate conservation laws}

Similarly, the well established theorem for constructing conservation laws taking advantage of the self-adjointness is now assumed to be valid up to the chosen order of the approximation using an approximate symmetry instead of a classical one.

We say that a vector field $C=\left(C^{1}, \cdots, C^{n}\right)$ is an approximate conserved current to the system (3) if

$$
\left.D_{i} C^{i}\right|_{F_{\alpha}^{0}+\nu F_{\alpha}^{1}=0}=\mathcal{O}\left(\nu^{2}\right) .
$$

For further and a deep discussion, see also $[18,19]$. 
Following Ibragimov's work, given a perturbed system (3), we can construct the adjoint perturbed system

$$
F_{\alpha}^{*}:=\frac{\delta\left(v^{\beta} F_{\beta}\right)}{\delta u^{\alpha}}=0 .
$$

Then, it is possible to construct an approximate conservation law for the system $F_{\alpha}=$ $0, F_{\alpha}^{*}=0$. However, such constructed vector is a nonlocal approximated vector for the perturbed system (3).

More recently, [23] introduced a rigorous basis relating approximate conservation laws and Ibragimov's theory on conservation laws, extending the results introduced in [15].

In fact, in [23] (see also [15]), it is introduced and discussed the following concept:

Definition 1. (Approximate nonlinear self-adjointness). The perturbed system (3) is called approximate nonlinear self-adjoint if the adjoint system (7) is approximate satisfied for all solutions $u$ of system (3) upon a substitution

$$
v^{\sigma}=\phi^{\sigma}(x, u)+\varepsilon \varphi^{\sigma}(x, u), \quad \sigma=1, \cdots, m,
$$

such that not all $\phi^{\sigma}$ and $\varphi^{\sigma}$ are identically equal to zero.

Once having this definition, we can construct local approximate conserved currents for the system under investigation by using the formula

$$
C^{i}=\xi^{i} \mathcal{L}+\sum_{i_{1}+\cdots+i_{n}=0}^{\infty} D_{i_{1}} \cdots D_{i_{n}}\left(W^{\alpha}\right) \frac{\delta^{*} \mathcal{L}}{\delta^{*} u_{i i_{1} \ldots i_{n}}^{\alpha}}
$$

were $W^{\alpha}=\eta^{\alpha}-\xi^{i} u_{i}^{\alpha}$,

$$
\frac{\delta^{*}}{\delta^{*} u_{i_{1} \ldots i_{n}}}=\frac{\partial}{\partial u}+\sum_{s=j_{1}+\cdots+j_{n}=1}^{\infty}(-1)^{s} \frac{\left(\begin{array}{c}
s \\
j_{1}, \ldots, j_{n}
\end{array}\right)}{\left(\begin{array}{c}
s+i_{1}+\cdots i_{n} \\
i_{1}+j_{1}, \ldots, i_{n}+j_{n}
\end{array}\right)} D_{j_{1}} \cdots D_{j_{n}} \frac{\partial}{\partial u_{\left(i_{1}+j_{1}\right) \ldots\left(i_{n}+j_{n}\right)}}
$$

$\left(\begin{array}{c}N \\ i_{1}, i_{2}, \ldots, i_{r}\end{array}\right)=\frac{N}{i_{1} ! i_{2} ! \ldots i_{r} !}, N=i_{1}+i_{2}+\cdots+i_{r}$ is the multinomial, $i_{j} \geq 0$ denotes the order of the derivative for the $j^{\text {th }}$ independent variable, $D_{i_{j}}=d^{i_{j}} / d\left(x^{j}\right)^{i_{j}}$ and $\mathcal{L}=v^{\beta} F_{\beta}$.

\section{Main results}

By using SYM interactively we obtain the approximate symmetries of equation (1):

$$
\begin{array}{lr}
\partial_{x}, & k \partial_{x}, \\
\partial_{t}, & k \partial_{t}, \\
k\left(t \partial_{t}-u \partial_{u}\right), & \left(t \partial_{t}-u \partial_{u}\right)-\frac{k}{2}\left(t \partial_{x}+\partial_{u}\right) .
\end{array}
$$

Comparing them with the Lie point symmetries of the unperturbed equation (2),

$$
\partial_{x}, \partial_{t}, t \partial_{t}-u \partial_{u}
$$

we can conclude that the perturbed equation inherits the symmetries of the unperturbed equation since all are stable. This fact can enable one to find a point transformation that links solutions of the unperturbed equation with the perturbed. Verily, the point transformation

$$
\tilde{x}=x+\frac{k}{2} t, \tilde{t}=t, \tilde{u}=u+\frac{k}{2}
$$

does exactly that. 
Now we turn to constructing the conservation law. First let the formal Lagrangian,

$$
\mathcal{L}=v(x, u)\left(u_{t}+k u_{x}-u_{t x x}+3 u u_{x}-2 u_{x} u_{x x}-u u_{x x x}\right) .
$$

By using it we obtain the adjoint equation,

$$
\left(3 u-u_{x x}+k\right) v_{x}+v_{t}=v_{x x x} u+v_{x x t}+v_{x x} u_{x} .
$$

By substituting $v=\phi(x, u)+k \varphi(x, u)$ into (10) and solving the resulted system we find that $\phi(x, u)=c_{1}+c_{2} u, \varphi(x, u)=c_{3}+c_{4} u$. Hence equation (1) is strictly self-adjoint in the approximate context. An expected outcome since equation (1) is strictly self-adjoint in the classical sense [12]. Putting now into the formula (8) the formal Lagrangian (9) with $v=u+k u$ and the only approximate symmetry of equation (1) that do not arise trivially from the classical symmetries of the unperturbed equation (2), $\left(t \partial_{t}-u \partial_{u}\right)-\frac{k}{2}\left(t \partial_{x}+\partial_{u}\right)$, we obtain the approximate conserved current:

$$
\begin{gathered}
C=\left(\frac { 1 } { 3 } k \left(3 u^{2}\left(6 t u_{t}-2 t u_{x x t}-6 u_{x x}+5\right)-u\left(3 t u_{t}\left(4 u_{x x}-3\right)+t u_{x x t}+12 u_{x t}+4 u_{x x}\right)+\right.\right. \\
\left.18 u^{3}+t u_{x} u_{x t}-t u_{t} u_{x x}-u_{x}^{2}\right)+2 u\left(u\left(-t u_{x x t}+3 t u_{t}-3 u_{x x}\right)+3 u^{2}-2\left(t u_{t} u_{x x}+u_{x t}\right)\right) \\
\frac{1}{3} k\left(u\left(-6 t u_{x x t}+6 t u_{t}+3\right)+6 u^{2}-t u_{x x t}+t u_{t}-2 t u_{x} u_{x x}+6 u_{x}^{2}-3 u_{x x}\right)+ \\
\left.2\left(t u\left(u_{t}-u_{x x t}\right)+u^{2}+u_{x}^{2}\right)\right)
\end{gathered}
$$

Verily,

$$
\left.D_{i} C^{i}\right|_{(1)}=-\frac{1}{3} k^{2}\left(2 u_{x}\left(9 u+3 t u_{t}+2\right)+t(6 u+1) u_{x t}\right) \approx 0
$$

\section{Concluding remarks}

In the present work a well known equation was seen under a different viewpoint: we assumed that the arbitrary parameter $k$ of the Camassa-Holm equation (1) takes only small values hence turning the equation into a perturbation problem. Having this as our starting point we found the approximate symmetries and through them an approximate conservation law was constructed.

Conservation laws are an invaluable asset that not only provide new non trivial analytic solutions but also can shed light to the stability and global behavior of the perturbed system and lead to the development of new numerical schemes. In the same way, approximate conservation laws can be an invaluable asset in the perturbation theory providing new numerical schemes and new approximate more dynamic solutions.

\section{References}

[1] V. A. Baikov, R. K. Gazizov and N. H. Ibragimov, Approximate symmetries, Matematicheskii Sbornik, 178 (1988) 435-450.

[2] G. W. Bluman and S. Kumei, "Symmetries and differential equations", Springer, New York, 1989.

[3] G. I. Burde, On the Use of the Lie Group Technique for Differential Equations with a Small Parameter: Approximate Solutions and Integrable Equations, Physics of Atomic Nuclei, 65 (2002) 990-995.

[4] R. Camassa and D. D. Holm, An Integrable Shallow Water Equation with Peaked Solitons, Phys. Rev. Lett., 71 (1993) 1661-1664. 
[5] R. Camassa, D. D. Holm and J. M. Hyman, A new integrable swallow water equation, Adv. Appl. Mech., 31 (1994) 1-33.

[6] S. Dimas and D. Tsoubelis, SYM: A new symmetry-finding package for Mathematica, In "The $10^{\text {th }}$ International Conference in MOdern GRoup ANalysis" (N. Ibragimov, C. Sophocleous and P. Damianou, eds.) pp. 64-70, University of Cyprus, Nicosia, 2005.

[7] A. S. Fokas, On a class of physically important integrable equations, Physica D, 87 (1995) $145-150$.

[8] B. Fuchssteiner, Some tricks from the symmetry-toolbox for nonlinear equations: generalizations of the Camassa-Holm equation, Physica D, 95 (1996) 229-243.

[9] W. I. Fushchich and W. M. Shtelen, On approximate symmetry and approximate solutions of the nonlinear wave equation with a small parameter, J. Phys. A: Math. Gen., 22 (1989) L887.

[10] D. D. Holm, J. E. Marsden and T. S. Ratiu, The Euler-Poincaré equations and semidirect products with applications to continuum theories, Adv. Math., 137 (1998) 1-81.

[11] P. E. Hydon, "Symmetry Methods for Differential Equations", Cambridge Texts in Applied Mathematics Cambridge University Press, Cambridge, $1^{\text {st }}$ ed., 2000.

[12] N. Ibragimov, R. S. Khamitova and A. Valenti, Self-adjointness of a generalized CamassaHolm equation, Appl. Math. Comp., 218 (2011).

[13] N. H. Ibragimov, "Transformation Groups Applied to Mathematical Physics", Mathematics and its Applications Springer, $1^{\text {st }}$ ed., 1985.

[14] N. H. Ibragimov, A new conservation theorem, J. Math. Anal. App, 333 (2007) 311-328.

[15] N. H. Ibragimov, Nonlinear self-adjointness in constructing conservation laws, In "Archives of ALGA" (N. H. Ibragimov, ed.), vol. 7/8 ALGA publications, Karlskrona, Sweden (20102011) pp. 1-90.

[16] N. H. Ibragimov, Nonlinear self-adjointness and conservation laws, J. Phys. A: Math. Theor., 44 (2011) 432002.

[17] N. H. Ibragimov and V. F. Kovalev, "Approximate and Renormgroup Symmetries", Nonlinear Physical Science Springer, $1^{\text {st }}$ ed., 2009.

[18] A. G. Johnpillai and A. H. Kara, Variational Formulation of Approximate Symmetries and Conservation Laws, Int. J. Theor. Phys., 40 (2001) 1501-1509.

[19] A. H. Kara, F. M. Mahomed and G. Ünal, Approximate Symmetries and Conservation Laws with Applications, Int. J. Theor. Phys., 38 (1999) 2389-2399.

[20] P. J. Olver, "Applications of Lie Groups to Differential Equations", vol. 107 of Graduate Texts in Mathematics, Springer, New York, $2^{\text {nd }}$ ed., 2000.

[21] L. Ovsiannikov, "Group Analysis of Differential Equations", Academic Press, $1^{\text {st }}$ ed., 1982, 432 pages.

[22] H. Stephani, "Differential Equations: Their Solution Using Symmetries", Cambridge University Press, Cambridge, $1^{\text {st }}$ ed., 1990, Editor: MacCallum, Malcolm.

[23] Z.-Y. Zhang, Approximate nonlinear self-adjointness and approximate conservation laws, J. Phys. A: Math. Theor., 46 (2013) pp13. 